\title{
Meiosis of triploid Lolium. III. Synaptonemal complex formation in the two inverse autoallotriploids of $L$. temulentum and L. multiflorum, TTM and TMM
}

\author{
HUW M. THOMAS \& BARRY J. THOMAS \\ Institute of Grassland and Environmental Research, Plas Gogerddan, Aberystwyth, Wales, SY23 3EB, U.K.
}

\begin{abstract}
The inverse types of autoallotriploid of Lolium temulentum $\times$ L. multiflorum have different trivalent frequencies and the possible causes were investigated by analysis of synaptonemal complex (SC) formation at zygotene. There is less synchrony in axial element (AE) formation in the TMM type than TTM. There are two autonomous pairing sites (APS) in the terminal regions, one at the end of the $\mathrm{AE}$ (telomeric) and one in a subterminal position. In the TMM combination, the L. temulentum subterminal APS cannot compete effectively with the pair of L. multiflorum APSs at synapsis and SCs are initiated between L. multiflorum AEs, but in TTM the $L$. multiflorum site competes with the pair of $L$. temulentum sites and SCs are frequently formed between homoeologous AEs. The L. temulentum chromosomes seem to be later in reaching a ready-to-pair stage and the results are taken to support the idea that genome asynchrony can lead to preferential pairing of chromosomes in polyploids.
\end{abstract}

Keywords: genome asynchrony, inverse autoallotriploids, Lolium, pairing initiation, synaptonemal complex.

\section{Introduction}

In the diploid hybrid between Lolium multiflorum and Festuca drymeja there is a barrier to pairing between the chromosomes of the two species that is not related to any lack of homologous sequences between the chromosomes (Thomas \& Morgan, 1990). It was considered that this barrier results from the large difference in DNA amount between the genomes, possibly reinforced by genes controlling chromosome pairing assumed to be present in $F$. drymeja. Morgan et al. (1986) analysed chromosome pairing at metaphase $I$ in six diploid hybrids of Festuca species and found that the greater the difference between the DNA content of the parental species, the more pronounced the failure of chromosome pairing in the $F_{1}$ hybrids. In Lolium, however, although $L$. temulentum has 50 per cent more DNA than L. multiflorum (Hutchinson et al., 1979), the chromosomes pair as seven bivalents in the diploid hybrid, and in some cases show little, if any, preferential pairing in the autoallotriploid with two sets of $L$. temulentum chromosomes and one of $L$. multiflorum (Evans \& Aung, 1985).
In the two inverse autoallotriploids of $L$. multiflorum (M) and L. temulentum (T) the difference in the DNA amounts of the genomes affects chromosome pairing and the effect is not symmetrical (Thomas, 1995). In the combination with two large genomes and one small genome (TTM) there is no evidence of preferential pairing between the homologous pairs, but in the combination with two small genomes and one large (TMM) there is preferential pairing between the L. multiflorum chromosomes, frequently excluding the $L$. temulentum chromosomes from the metaphase I configurations at a high frequency. The differences between the two autoallotriploid types manifest themselves most clearly in their respective trivalent frequencies: mean trivalents per cell $=5.27$ in TTM and 2.06 in TMM. The difference partly results from chiasma frequency, but there is still more than a two-fold difference when the effect of chiasmata is removed.

Comparisons of the TTM autoallotriploid and the autotriploids of the two species (i.e. MMM and TTT) showed that the differences in trivalent frequencies are dependent only on chiasma numbers (Thomas, 1995). With fewer chiasmata in TTM 
autoallotriploids than the autotriploids, fewer prophase I trivalents are maintained to metaphase I. These results imply that in the TTM triploids, prophase I trivalent frequency is the same as in the autotriploids, as is the number and position of the pairing partner exchanges (PPEs). In TMM triploids, where the reduced frequency of trivalents is only partly explained by chiasma frequency, the number and/or position of PPEs must be different from the autotriploids and TTM autoallotriploids.

Synaptonemal complex formation was studied in the autoallotriploid $L$. temulentum $(2 \times) \times L$. perenne $(4 \times)$ by serial sectioning (Jenkins, 1985). One early zygotene nucleus was analysed plus the $L$. perenne complement of one pachytene nucleus. The genotype studied involved the low-pairing genotype (Lp10) of L. perenne and had two B chromosomes. The combined effect of the Lp10 genotype and the B chromosomes suppresses homoeologous pairing and seven $L$. perenne bivalents are found at metaphase I. Jenkins (1985) also found seven bivalents in the pachytene nucleus but one trivalent in the zygotene nucleus. The present study deals with the synaptonemal complex analyses of the two autoallotriploids L. temulentum $(4 \times) \times L$. multiflorum $(2 \times)=$ TTM and $L$. temulentum $(2 \times) \times L$. multiflorum $(4 \times)=$ TMM. There are no major pairing control genes in the accessions of either species.

\section{Materials and methods}

The inverse autoallotriploids are hybrids between diploid $L$. temulentum and tetraploid $L$. multiflorum (TMM), and tetraploid L. temulentum and diploid $L$. multiflorum (TTM). Thomas (1995) gives details of the hybrids and Thomas (1990) describes the methods for spreading and staining SCs. The silver stained SCs were examined on a Jeol 100S Transmission Electron Microscope and electron micrographs were photo-enlarged to a final magnification of 9000 . Measurements were made with a digitizer tablet and microcomputer.

\section{Results}

\section{General pattern of synapsis}

Table 1 summarizes the analysis of SC formation in 17 prophase I nuclei of the TTM autoallotriploids. The total axial element (AE) length ranges from $1031 \mu \mathrm{m}$ to $691 \mu \mathrm{m}$ and the pairing varies between 17 and 78 per cent. There is a significant negative correlation between the $\mathrm{AE}$ length of each nucleus and pairing as a percentage, transformed to angles $(r=-0.674 ; P<0.01)$. The nuclei are arranged in an increasing order of pairing which probably represents a rough order of stage, that is, the nucleus with 17 per cent of the length paired is at zygotene while nucleus 16 with 67 per cent of AE length paired, including extensive heterologous pairing, can be considered to be at pachytene. However, the transition between zygotene and pachytene cannot be judged because there are heterologously paired AEs in nuclei where homologous or homoeologous pairing has, it appears, not reached its possible maximum.

In the trivalents in autotriploid $L$. multiflorum (Thomas \& Thomas, 1994) the unpaired regions of $\mathrm{AE}$ were consistently longer than the equivalent AEs incorporated into SCs, and within each trivalent, the shortest $\mathrm{AE}$ was the one that was most paired. In six out of 10 trivalents that were fully analysed in TTM, the shortest AE is the least paired. It is likely therefore that the difference in AE lengths in these trivalents reflects the difference in the chromosome sizes of the two species and the shortest $\mathrm{AE}$ is of L. multiflorum. However, in four trivalents the shortest $\mathrm{AE}$ is also the most paired as expected if the three chromosomes had been of equal length. The $L$. multiflorum chromosome cannot therefore be identified in these cases. In the

Table 1 A summary of the analysis of synaptonemal complex formation in 17 nuclei from four genotypes of the autoallotriploid TTM

\begin{tabular}{rccc}
\hline $\begin{array}{c}\text { Nucleus } \\
\text { number }\end{array}$ & $\begin{array}{c}\text { SC pairing } \\
(\%)\end{array}$ & $\begin{array}{c}\text { Triple associations } \\
(\%)\end{array}$ & $\begin{array}{c}\text { Axial element } \\
\text { length }(\mu \mathrm{m})\end{array}$ \\
\hline 1 & 17 & 0 & 929 \\
2 & 26 & 0 & 1031 \\
3 & 37 & 0 & 947 \\
4 & 40 & 0.8 & 1004 \\
$* 5$ & 47 & 0 & 856 \\
6 & 48 & 0 & 834 \\
7 & 51 & 4.0 & 915 \\
$\dagger 8$ & 51 & 0 & 822 \\
9 & 55 & 0 & 874 \\
10 & 55 & 0.6 & 787 \\
$\dagger 11$ & 57 & 0.3 & 948 \\
12 & 59 & 0 & 952 \\
$\dagger 13$ & 59 & 2.3 & 891 \\
14 & 59 & 1.0 & 843 \\
$\dagger 15$ & 60 & 0 & 805 \\
$\ddagger 16$ & 67 & 0.5 & $?$ \\
$* 17$ & 78 & 0 & 691 \\
\hline
\end{tabular}

$\ddagger$ Genotype A1, * genotype A2, † genotype A11. Other nuclei from genotype A5. ? Magnification not known. 
six trivalents where homologous and homoeologous pairing can be distinguished, five telomeric SCs involve two $L$. temulentum AEs and seven telomeric SCs have one L. temulentum and one L. multiflorum AE synapsed: in this limited sample there is no indication of a preference for homologous pairing adjacent to the telomeres. A total of 12 bivalents was recorded, a mean of 0.71 per cell, with a maximum of three in one cell.

There is intimate association of three AEs in seven of the nuclei (Table 1). Ten of the 14 triple associations are in intercalary regions and four are at the telomeres. Most of the triple associations are clearly not triple synapses, i.e. three AEs joined by two central regions, but represent the close alignment of AEs prior to SC formation (cf. autotriploid L. multiflorum, Thomas, 1995). However, some are inconclusive.

Table 2 summarizes the analysis of SC formation in 14 prophase I nuclei of the TMM autoallotriploid. The total AE length ranges from $1215 \mu \mathrm{m}$ to $690 \mu \mathrm{m}$ and the pairing varies between 10 and 74 per cent. There is a highly significant negative correlation between the AE length of each nucleus and pairing as a percentage, transformed to angles $(r=-0.889$; $P<0.001)$. The nuclei are again arranged in increasing order of pairing which probably gives a rough order of stage.

In the earliest of these nuclei, besides the SCs, 15 per cent of the total $\mathrm{AE}$ length is aligned as pairs of $\mathrm{AEs}$ and 2 per cent of the AE length is aligned as triples. The most obvious feature of the AEs in the

Table 2 A summary of the analysis of synaptonemal complex formation in 14 nuclei from a single genotype of the autoallotriploid TMM

\begin{tabular}{lccc}
\hline $\begin{array}{l}\text { Nucleus } \\
\text { number }\end{array}$ & $\begin{array}{c}\text { SC pairing } \\
(\%)\end{array}$ & $\begin{array}{c}\text { Triple associations } \\
(\%)\end{array}$ & $\begin{array}{c}\text { Axial element } \\
\text { length }(\mu \mathrm{m})\end{array}$ \\
\hline 1 & 10 & 0 & 1190 \\
2 & 22 & 0 & 1215 \\
3 & 25 & 0 & 1059 \\
4 & 30 & 0 & 1092 \\
5 & 45 & 0 & 783 \\
6 & 45 & 0 & 775 \\
7 & 46 & 0 & 932 \\
8 & 50 & 1.7 & 912 \\
9 & 50 & 2.4 & 835 \\
10 & 53 & 0 & 950 \\
11 & 63 & 0 & 711 \\
12 & 64 & 0 & 838 \\
13 & 67 & 0 & 690 \\
14 & 74 & 0 & 734 \\
\hline
\end{tabular}

early nuclei, however, is their discontinuous nature. In addition to the unpaired AEs recorded as extensions of SCs, and aligned AEs, there are more than 70 fragments of AEs in nucleus 1 that make up 70 per cent of the total AE length. This is a feature seen in most of the TMM spreads, the exceptions being the nuclei at later stages where all AEs appear quite distinct. In common with the pachytene TTM nuclei, there is extensive heterologous pairing although there are also unpaired AEs.

Few configurations could be fully analysed in the TMM nuclei but in one trivalent, where the three AEs were traced from telomere to telomere, the two shorter AEs are $30 \mu \mathrm{m}$ and $32 \mu \mathrm{m}$ long and 97 and 77 per cent paired, respectively. The third axis is $49 \mu \mathrm{m}$ long and 11 per cent paired (Fig. 1). If the longer $\mathrm{AE}$ is the L. temulentum chromosome then both telomeres of the L. multiflorum AEs are homologously paired. There was a mean of 1.2 bivalents per nucleus, the maximum being three.

\section{Pairing at the telomeres}

It has not been possible to recognize all the telomeres. In analysing telomeric features therefore it is difficult to quantify the observations. In the TTM nuclei, on average, 11 sets of paired telomeres were identified per nucleus. One in six of these are associations of three AEs; either the third telomere is associated end to end with one of the two paired telomeres (Fig. 2a) or it is closely aligned with the SC (Fig. 2b,c), or all three AEs are equally closely bound and in some instances resemble triple synapses, i.e. they may be held together by two central regions (Fig. 2d). In one case a parallel arrangement of three AEs is just $0.4 \mu \mathrm{m}$ long with the 'third' $\mathrm{AE}$ in the middle while the SC that extends from the telomere is formed by the two outer AEs (Fig. 2e). The AEs may be mismatched at the telomere and in each case recorded an SC is formed between the shortest $\mathrm{AE}$ and one of the longer AEs (Fig. 2f-h). The two longer AEs may form a short SC that is then replaced by an SC between one of these AEs and the shorter AE (Fig. 2d, i). In TMM nuclei, on average, eight paired sets of telomeres were identified per nucleus - no association of three AEs of any description was found at the telomeres.

PPEs often occur in subtelomeric regions in TTM nuclei (Fig. 3a-d) and in TMM nuclei (Fig. 3e,f). There does, however, appear to be a quantitative difference between the two triploid types. In TTM there is a PPE within $10 \mu \mathrm{m}$ of the telomere in 44 of the 151 telomere groups recorded, 32 PPEs are within $4 \mu \mathrm{m}$ of the telomere with 12 in the subse- 

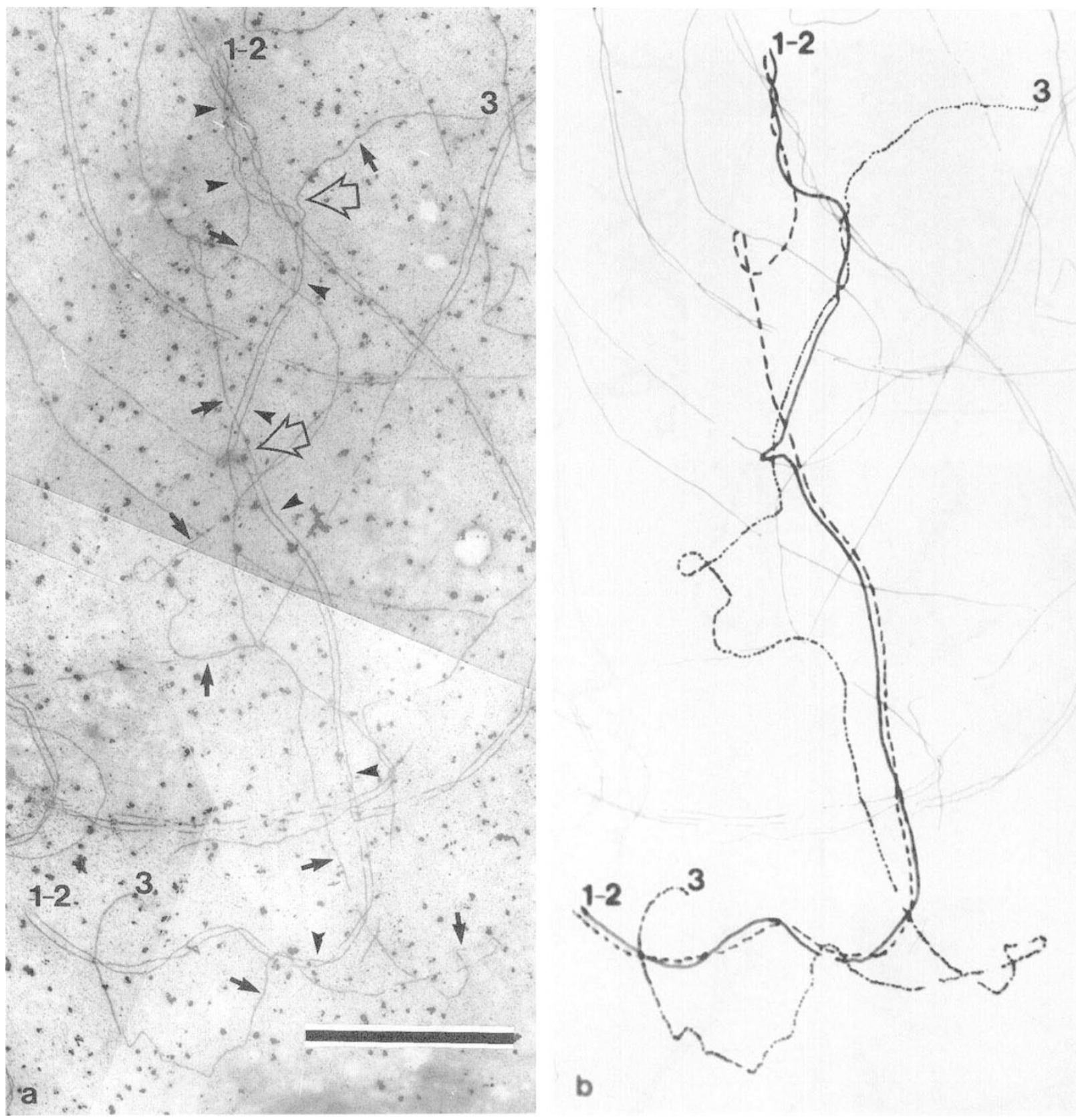

Fig. 1 (a) A trivalent from TMM nucleus 8 . Axes 1 and 2 are $30 \mu \mathrm{m}$ and $32 \mu \mathrm{m}$ long and 97 and 77 per cent paired, respectively; axis 3 is $49 \mu \mathrm{m}$ long and 11 per cent paired. SCs marked by arrowheads, unpaired AEs by small arrows, PPEs by large open arrows. Bar represents $5 \mu \mathrm{m}$. (b) Interpretative drawing of (a).

quent $6 \mu \mathrm{m}$. The number of PPEs in these two regions is highly significantly different from $4: 6$ $\left(\chi_{1}^{2}=18.296 ; P<0.001\right)$. In the TMM autoallotriploids, a PPE is present within $10 \mu \mathrm{m}$ of the telomere in only 12 of the 111 telomeres recorded, six PPEs are within the first $4 \mu \mathrm{m}$ and six in the subsequent $6 \mu \mathrm{m}$. The number of subtelomeric PPEs recorded in each autoallotriploid type, adjusted to 100 telo- meres, is presented graphically in Fig. 4. Clearly, there is a much higher incidence of PPEs near to the TTM telomeres than the TMM telomeres, but in both TTM and TMM the highest incidence of PPEs occurs between 1 and $2 \mu \mathrm{m}$ of the telomere. The frequency of PPEs within $10 \mu \mathrm{m}$ of the telomere in autotriploid L. multiflorum is shown for comparison. Again the highest incidence of PPEs is between 1 

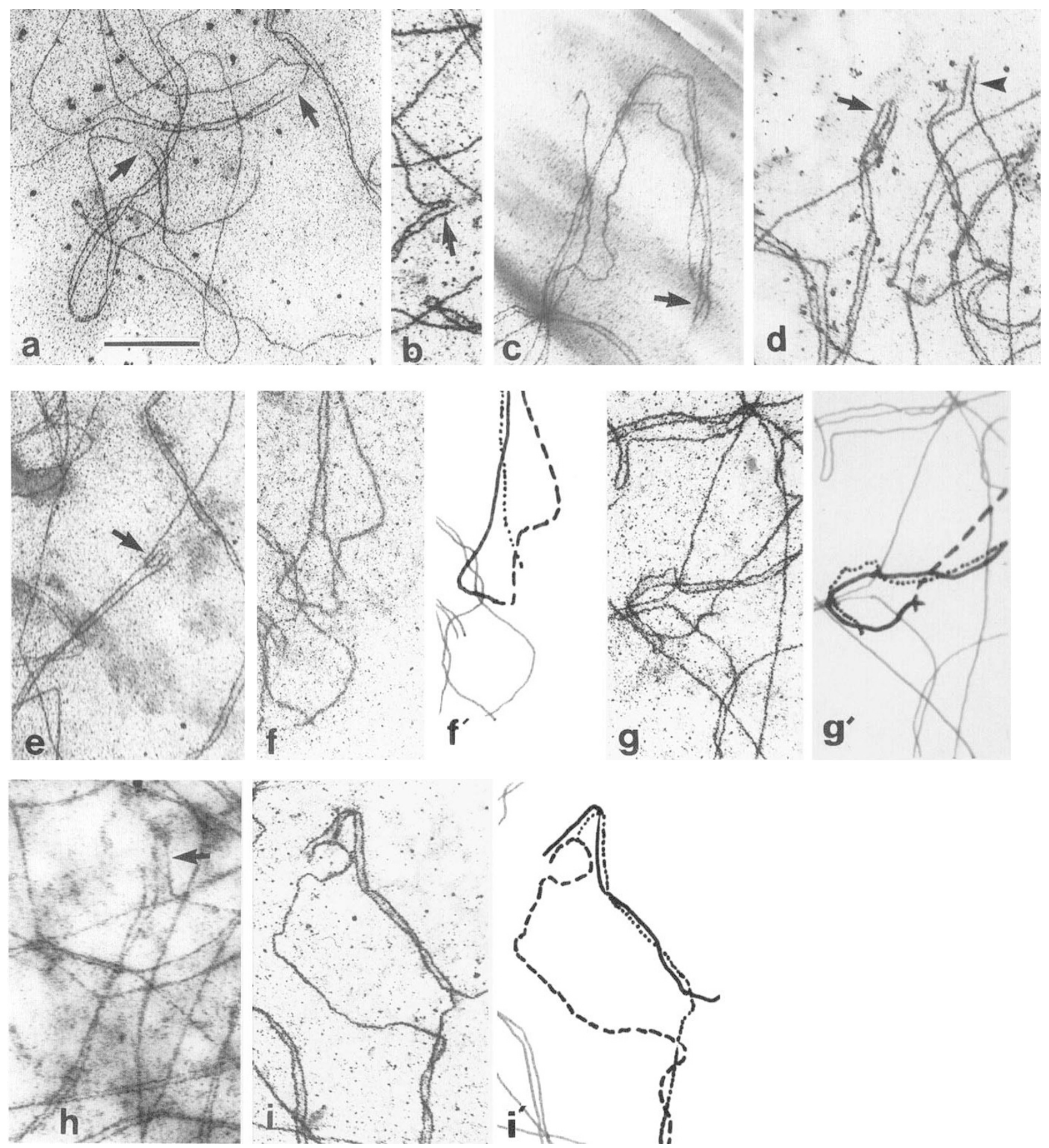

Fig. 2 Telomere associations in TTM. (a) End-to-end (arrows); (b,c) side-on (arrows); (d) side-on, may be double SC (arrow), also SC between longer AEs replaced by SC involving shorter AE (arrowhead); (e) side-on with SC formed between the two outer AEs; $(f, g)$ associations of unsynapsed longer AEs but SC involves shorter AE; $\left(f^{\prime}, g^{\prime}\right)$ interpretative drawings; (h) side-on, SC involves shorter AE; (i) SC between longer AE replaced by SC involving shorter AE; (i') interpretative drawing. Bar represents $2 \mu \mathrm{m}$ and applies to all except (h). 
Fig. 3 Subtelomeric PPEs (arrows) in TMM (a-d) and TMM (e,f). Bar represents $2 \mu \mathrm{m}$.

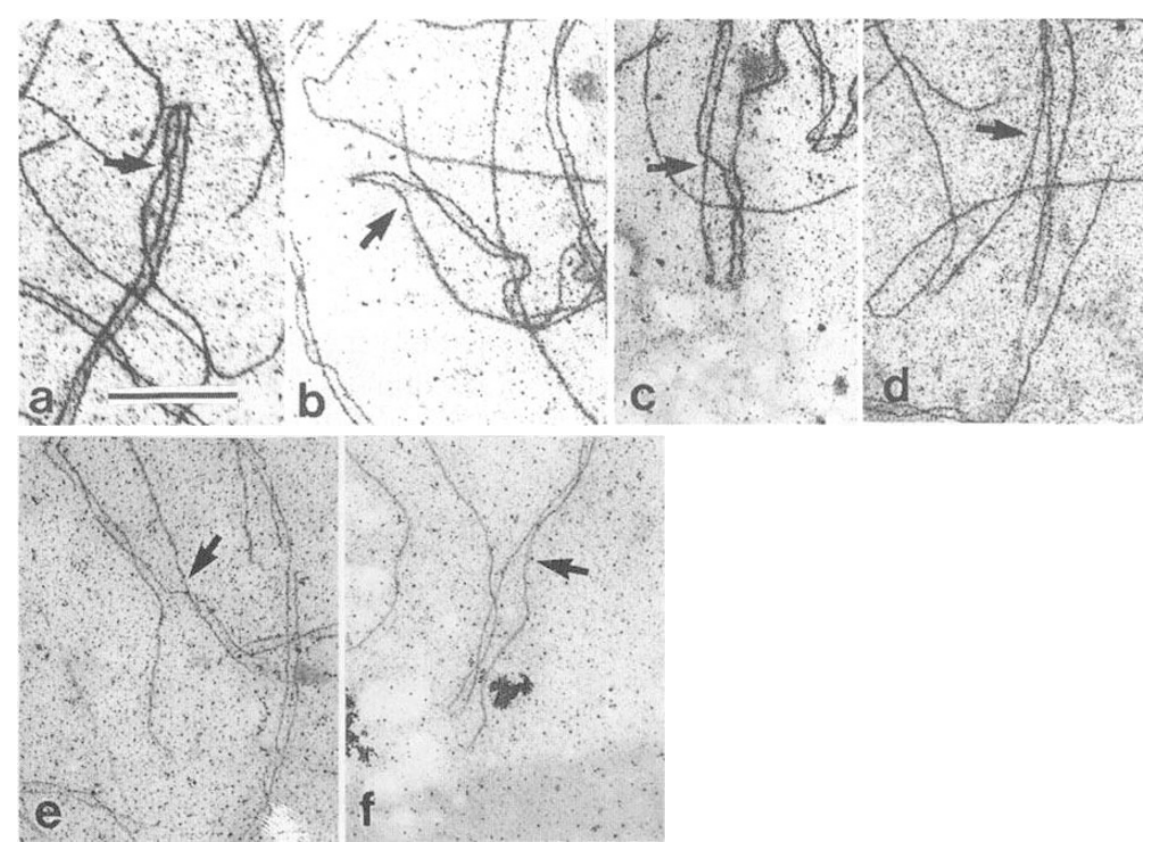

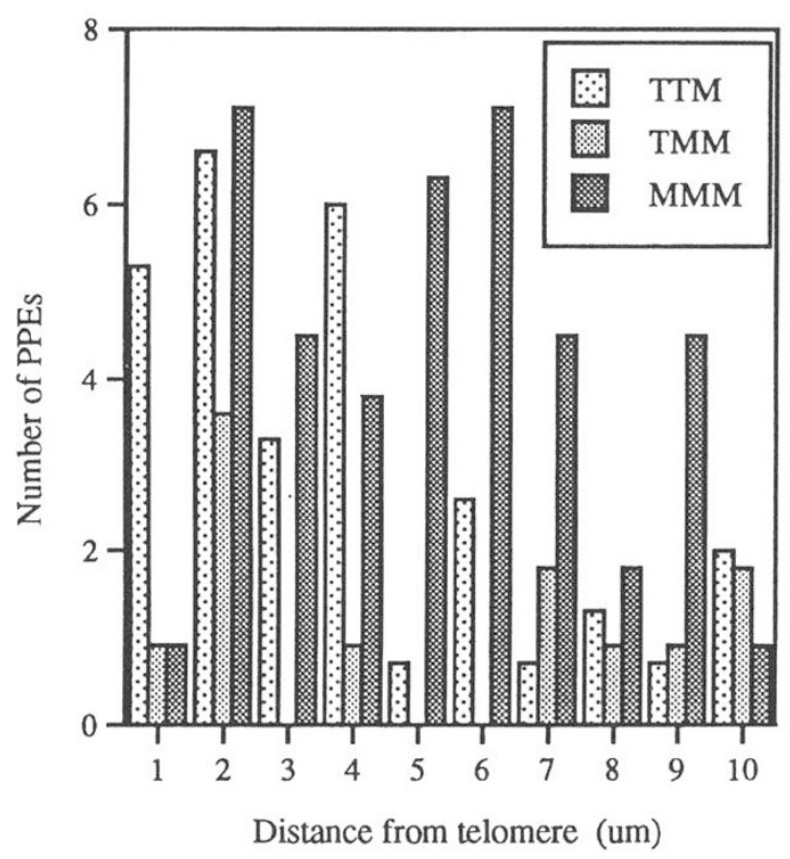

Fig. 4 The frequencies and positions of PPEs in subtelomeric regions adjusted as per 100 telomeres in the two autoallotriploids TTM and TMM and autotriploid $L$. multiflorum, MMM. and $2 \mu \mathrm{m}$ of the telomere but there is not a greater occurrence of PPEs within the first $4 \mu \mathrm{m}$ than the following $6 \mu \mathrm{m}$.

Most of the SCs in the TMM nuclei are in telomeric regions. In TMM nucleus 5, all 14 paired telomeres were identified and the extent of pairing adjacent to each telomere was different. There were two fully formed bivalents, $27 \mu \mathrm{m}$ and $29 \mu \mathrm{m}$ long while the SCs extending from the other telomeres were between $1.4 \mu \mathrm{m}$ and $19 \mu \mathrm{m}$ long. Four other SC regions in this nucleus not in telomeric regions ranged from $4 \mu \mathrm{m}$ to $9 \mu \mathrm{m}$ in length. All the paired AEs and the unpaired AEs extending from the SC together with a pair of aligned AEs made up 62 per cent of the total length of the nucleus. The remaining 38 per cent consisted of 50 fragments of AE.

\section{Discussion}

Analyses of chromosome configurations at metaphase I showed a large difference between the two inverse types of autoallotriploid, TTM and TMM, in the way the chromosomes had paired. As most of the difference was independent of chiasma frequency the conclusion was reached that the initiation of pairing must be different in the two autoallotriploids (Thomas, 1995). The lower trivalent frequency in the TMM plants and a high frequency of ring bivalents indicates that synapsis in the distal 
regions is between homologous chromosomes. This means that in most trisomes at prophase I, either the two L. multiflorum axial elements pair as a bivalent, or homoeologous synapsis is restricted to the more median parts of the trivalent and flanked by a pair of PPEs. Such a trivalent is retained to metaphase I only when a chiasma occurs in the homoeologous median SC, otherwise chiasmata in only the subtelomeric homologous SCs means a bivalent is found at metaphase I.

Given the number of nuclei analysed at prophase $\mathrm{I}$, it is not possible to tell if bivalents are formed at a higher frequency in TMM than in TTM (although more bivalents were found in the former: 1.2 bivalents per nucleus in TMM and 0.7 in TTM). Furthermore, it is not possible to determine the number of PPEs in the TMM trivalents or see if they conform to the type of trivalent described above because few configurations in the TMM spreads are complete. However, the trivalent shown in Fig. 1 does conform to this model.

One proposed means of ensuring that pairing is initiated between homologues is that the telomeres of homologous chromosomes are attached to the same point on the membrane, or move to the same point on the membrane prior to the onset of synapsis (reviewed by Loidl, 1990). If that is the case, association of all three homologous telomeres in autotriploid $L$. multiflorum may be a residual effect of that attachment (Thomas \& Thomas, 1994) and, by the same token, the association of telomeres in TTM indicates that these telomeres are also attached together to the nuclear membrane. However, no telomeric associations were found between two L. multiflorum and one L. temulentum telomeres in TMM. In human spermatocyte nuclei at the leptotene-zygotene transition, homologous telomeres are still randomly distributed and even at late zygotene, unpaired telomeres are widely separated on the nuclear envelope (Rasmussen \& Holm, 1980). If the telomeres move together just prior to synapsis, then in TMM, the L. temulentum telomere may not have had sufficient time to move.

Rather than a symptom of possible attachment to the nuclear envelope, the association of telomeres may be a mechanism that acts directly between telomeres and is part of the homologue recognition mechanism; in which case the mechanism is not (yet) active in both the L. temulentum and L. multiflorum telomeres in the TMM combination but is active in the telomeres of the three genomes in the TTM autoallotriploid.

An autonomous pairing site (APS) is considered to be a site capable of initiating pairing between any two of three or more chromosomes and therefore generating a PPE (Callow \& Gladwell, 1984). The presence of PPEs close to the telomere means there must be more than one APS in the telomeric and subtelomeric regions in some chromosomes. One APS must be within $0.4 \mu \mathrm{m}$ of the telomere in at least one trisome. For a PPE to occur, two APSs must have synapsed two different AEs, therefore three AEs must have been aligned prior to synapsis (cf. Thomas \& Thomas, 1994). This happens in both TTM and TMM, as subtelomeric PPEs are seen in both, but occurs more frequently in TTM where subtelomeric PPEs are more common. There have been many reports of the initiation of pairing near the telomeres, e.g. Jones \& Croft (1986). In Allium, pairing usually starts at subterminal sites and the telomeric regions are among the last to pair (Albini \& Jones, 1987). Rasmussen (1986) identified two types of association in male Bombyx that preceded $\mathrm{SC}$ formation, the first was between subtelomeric regions, the other between the telomere attachment plaques. This also seems to be the case in Lolium.

In the L. multiflorum AEs, when the subtelomeric APS is within about $1 \mu \mathrm{m}$ of the telomeric APS, the two sites act in concert with the result that only one PPE was recorded within $1 \mu \mathrm{m}$ of the telomere in MMM and only one in TMM (Fig. 4). When the distance between the APSs is greater than $1 \mu \mathrm{m}$, they act independently explaining the relatively high incidence of the PPEs between 1 and $2 \mu \mathrm{m}$ of the telomere in all of the genome combinations.

In some trisomes, the distance between the subtelomeric APS and the telomere may be greater in the L. temulentum $\mathrm{AE}$ than in the L. multiflorum $\mathrm{AE}$ (Fig. 2f,g,i). In TTM, although the L. multiflorum AE can compete effectively at the subtelomeric APS the $\mathrm{AE}$ is too short to reach the L. temulentum telomere and the two L. temulentum telomeric APSs pair preferentially. This leads to a disproportionately high frequency of PPEs within $1 \mu \mathrm{m}$ of the telomere in TTM. However, most chiasmata presumably occur at, or proximal to, the subtelomeric APS and therefore there is no evidence of preferential pairing at metaphase I (Thomas, 1995).

In nucleus 5 of TMM, pairing was complete in two bivalents while two telomeres had SCs of less than $2 \mu \mathrm{m}$. If this is generally the case in TMM nuclei, the asynchrony between L. multiflorum and L. temulentum telomeric regions could be sufficient in some trisomes to guarantee bivalent pairing between $L$. multiflorum homologues; in other trisomes the asynchrony may be sufficient only in ensuring that terminal regions are homologously paired, while the asynchrony may be insufficient to 
prevent homoeologous pairing in more median regions; in yet other trisomes the pairing at the $L$. multiflorum telomeric regions may be sufficiently late as to allow the L. temulentum AE to compete at the telomeric or subtelomeric APSs. There are reports of asynchrony between bivalents in lily (Holm, 1977), Bombyx (Rasmussen, 1976, 1986) and humans (Rasmussen \& Holm, 1980). In humans there were fully paired bivalents and totally unpaired AEs in the same nucleus. A greater asynchrony between homoeologues within some trisomes than others can explain why $\mathbf{M}-\mathbf{M}$ preferential pairing is not equally expressed in all the TMM trisomes (Thomas, 1995).

The duration of meiosis is correlated with DNA amount (Bennett, 1976). As L. temulentum has 50 per cent more DNA than $L$. multiflorum, the normal duration of meiosis can be assumed therefore to be longer in $L$. temulentum than in $L$. multiflonum. In the TMM autoallotriploid the $L$. temulentum genome may have insufficient time to compete on equal terms for all the APSs allowing the L. multiflorum chromosomes to pair preferentially. On the other hand, in TTM, if the haploid set of $L$. multiflorum chromosomes reaches the state of readiness to pair before the L. temulentum chromosomes then when the $L$. temulentum chromosomes are ready to pair, the $L$. multiflorum chromosomes are able to compete at all APSs. Indeed, where length differences were seen between AEs at the telomeres, the shorter AE, presumably L. multiflorum, was involved in the subterminal SC in every case.

Thomas (1988) proposed that genome asynchrony, evident at metaphase $I$ in the tetraploid hybrid Hordeum vulgare $\times H$. bulbosum, would, if present at zygotene, be responsible for the reduced homoeologous pairing. The findings presented here support the proposal that asynchrony between homoeologous chromosomes at pairing initiation may be responsible for preferential pairing of homologous chromosomes.

\section{References}

ALBINI, S. M. AND JONES, G. H. 1987. Synaptonemal complex spreading in Allium cepa and A. fistulosum I. The initiation and sequence of pairing. Chromosoma, 95, 324-338.

BENNETT, M. D. 1976. The cell in sporogenesis and spore development. In: Yeoman, M. M. (ed.) Cell Division in
Higher Plants, pp. 161-198. Academic Press, London. CALlOW, R. S. AND GLADWELl, I. 1984. A general treatment of chromosome synapsis in even-numbered polyploids. J. Theor. Biol., 106, 455-494.

EVANS, G. M. AND AUNG, T. 1985. Identification of a diploidizing genotype of Lolium multiflorum. Can. J. Genet. Cytol., 27, 498-505.

HOLM, Р. в. 1977. Three dimensional reconstruction of chromosome pairing during the zygotene stage of meiosis in Lilium longiflorum (Thumb). Carlsberg Res. Comm., 42, 103-151.

HUTCHINSON, J., REES, H. AND SEAL, A. G. 1979. An assay of activity of supplementary DNA in Lolium. Heredity, $\mathbf{4 3}$, 411-421.

JENKINS, G. 1985. Synaptonemal complex formation in hybrids of Lolium temulentum $\times$ Lolium perenne (L.) II. Triploid. Chromosoma, 92, 387-390.

JONES, G. H. AND CROFT, J. A. 1986. Surface spreading of synaptonemal complexes in locusts. II. Zygotene pairing behaviour. Chromosoma , 93, 489-495.

LOIDL, J. 1990. The initiation of meiotic chromosome pairing: the cytological view. Genome, 33, 759-778.

MORGAN, w. G., THOMAS, H., EVANS, M. AND BORRILL, M. 1986. Cytogenetic studies of interspecific hybrids between diploid species of Festuca. Can. J. Genet. Cytol., 28, 921-925.

RASMUSSEN, s. w. 1976. The meiotic prophase in Bombyx mori females analyzed by three dimensional reconstructions of synaptonemal complexes. Chromosoma, 54, 245-293.

RASMUSSEN, S. w. 1986. Initiation of synapsis and interlocking of chromosomes during zygotene in Bombyx spermatocytes. Carlsberg Res. Comm., 51, 401-432.

RASMUSSEN, S. W. AND HOLM, P. B. 1980. Mechanics of meiosis. Hereditas, 93, 187-216.

тномаs, н. м. 1988. Chromosome elimination and chromosome pairing in tetraploid hybrids of Hordeum vulgare $\times$ H. bulbosum. Theor. Appl. Genet., 76, 118-124.

THOMAS, H. M. 1990. Analysis of synaptonemal complexes in the amphiploid of Lolium multiflorum $\times$ Festuca drymeja. Genome, 33, 903-907.

THOMAS, H. M. 1995. Meiosis in triploid Lolium. II. Discrepancies between the analyses of chromosome configurations at metaphase I in inverse autoallotriploid combinations. Heredity, 75, 446-452.

THOMAS, H. M. AND MORGAN, w. G. 1990. Analysis of synaptonemal complexes and chromosome pairing at metaphase I in the diploid intergeneric hybrid Lolium multiflorum $\times$ Festuca drymeja. Genome, 33, 465-471.

THOMAS, H. M. AND THOMAs, B. J. 1994. Meiosis in triploid Lolium. I. Synaptonemal complex formation and chromosome configuration at metaphase $I$ in aneuploid autotriploid L. multiflorum. Genome, 37, 181-189. 